\title{
Disappearance of Spontaneous Echographic Contrast after Balloon Mitral Valvuloplasty: An Indicator of Sustained Hemodynamic Improvement
}

\author{
TON SLAGBOOM, M.D., MEINDERT TAAMS, M.D., WIM D. VLETTER, M.SC., \\ GEORGE SUTHERLAND, M.D., PH.D., PIM J. DE FEYTER, M.D., PH.D., \\ and PATRICK W. SERRUYS, M.D., PH.D.
}

From the Thoraxcenter, Department of Cardiology, Erasmus University, Rotterdam, The Netherlands

\begin{abstract}
In three patients undergoing mitral balloon valvuloplasty for mitral stenosis transesophageal echocardiography was performed before, immediately after, and 6 months after the procedure. In the one patient with persistent hemodynamically favorable result, the spontaneous echocardiographic contrast, which was seen in all three preoperatively, did not recur; in the other two patients the phenomenon was observed again after 6 months. We conclude that the disappearance of spontaneous echocardiographic contrast might be a functional morphological measure of sustained hemodynamic improvement after balloon mitral valvuloplasty. (J Interven Cardiol 1990:3:2)
\end{abstract}

\section{Introduction}

Transesophageal echocardiography is a sensitive technique to examine morphological structures in the left atrium. It has a greater sensitivity for the detection of thrombi in the left atrial appendage than precordial echocardiography. ${ }^{1}$ Spontaneous echographic contrast detected by transesophageal echocardiography is an indicator for an increased thromboembolic risk in patients with mitral stenosis and atrial fibrillation. The effect changes in transmitral flow after percutaneous transluminal mitral valvuloplasty on spontaneous echographic contrast has not previously been described. This article reports the features of spontaneous contrast after balloon mitral valvuloplasty and its relationship with transmitral flow.

Address for reprints: P.W. Serruys, Thoraxcenter, Catheterization Laboratory, Erasmus University, P.0. Box 1738, 3000 DR Rotterdam, The Netherlands.

\section{Patients}

Three women, aged 33, 44, and 49 years, with severe rheumatic mitral stenosis and mild or no mitral regurgitation and without accompanying valvular disease, were selected for balloon valvuloplasty. Previously no one had evidence of arterial thromboembolic events. Patient characteristics are shown in Table I.

Precordial two-dimensional echocardiography with a $3.5-\mathrm{MHz}$ transducer showed an enlarged left atrium, a thickened mitral valve, and fusion of the commissures in all patients. Transesophageal echocardiography was performed, using a $5.6-\mathrm{MHz}$ transducer (64 elements) mounted at the tip of gastroscope and interfaced with a Hewlett-Packard ultrasonograph (HP 77020 AC [Hewlett-Packard, Andover, MA, USA]). There was no evidence of left atrial thrombus. Spontaneous echographic contrast, swirling in phase with the inflow of blood from the pulmonary veins, ${ }^{2}$ filling the entire enlarged atrium, was noted in all three (Fig. 1). 


\section{SLAGBOOM, ET AL.}

Table I. Catheterization Measurements and Echocardiographic Observations Before, Immediately After and 6 Months After Mitral Valvuloplasty

\begin{tabular}{|c|c|c|c|c|c|c|c|c|c|}
\hline \multirow[b]{2}{*}{ Patient } & \multicolumn{3}{|c|}{1} & \multicolumn{3}{|c|}{2} & \multicolumn{3}{|c|}{3} \\
\hline & B & A & $6 \mathrm{~m}$ & B & A & $6 \mathrm{~m}$ & B & A & $6 \mathrm{~m}$ \\
\hline Rhythm & & SR & & & $\mathrm{AF}$ & & & SR & \\
\hline LA $\operatorname{dim}(\mathrm{mm})$ & 51 & & 60 & 60 & & 65 & 48 & & 60 \\
\hline Sp Contr & + & & - & + & & + & + & & + \\
\hline Gradient MV (mmHg) & 17 & 6 & 11 & 16 & 6 & 12 & 21 & 7 & 14 \\
\hline $\mathrm{CO}(\mathrm{L} / \mathrm{min})$ & 4.8 & 4.1 & 4.6 & 5.8 & 4.3 & 3.9 & 3.0 & 3.9 & 3.7 \\
\hline Flow $(\mathrm{mL} / \mathrm{sec})$ & 148 & 170 & 170 & 160 & 263 & 195 & 122 & 170 & 122 \\
\hline $\mathrm{MV}$ area $\left(\mathrm{cm}^{2}\right)$ & 1.2 & 2.3 & 1.7 & 1.3 & 3.6 & 2.0 & 0.9 & 2.1 & 1.1 \\
\hline MR & $2 / 4$ & $1 / 4$ & $2 / 4$ & $1 / 4$ & $1 / 4$ & $1 / 4$ & 0 & 0 & 0 \\
\hline
\end{tabular}

B, A = immediately before and after valvuloplasty; $6 \mathrm{~m}=$ values 6 months after valvuloplasty; $L A$ dim = precordial echocardiographic dimension of the left atrium; Sp Contr = spontaneous echographic contrast. MV Area = calculated mitral valve area during catheterization by use of Gorlin formula; Gradient $\mathrm{MV}=$ mean transmitral diastolic pressure gradient at catheterization; $\mathrm{CO}=$ cardiac output measured by thermodilution; Flow = calculated diastolic transmitral flow; MR = angiographic severity of mitral regurgitation.

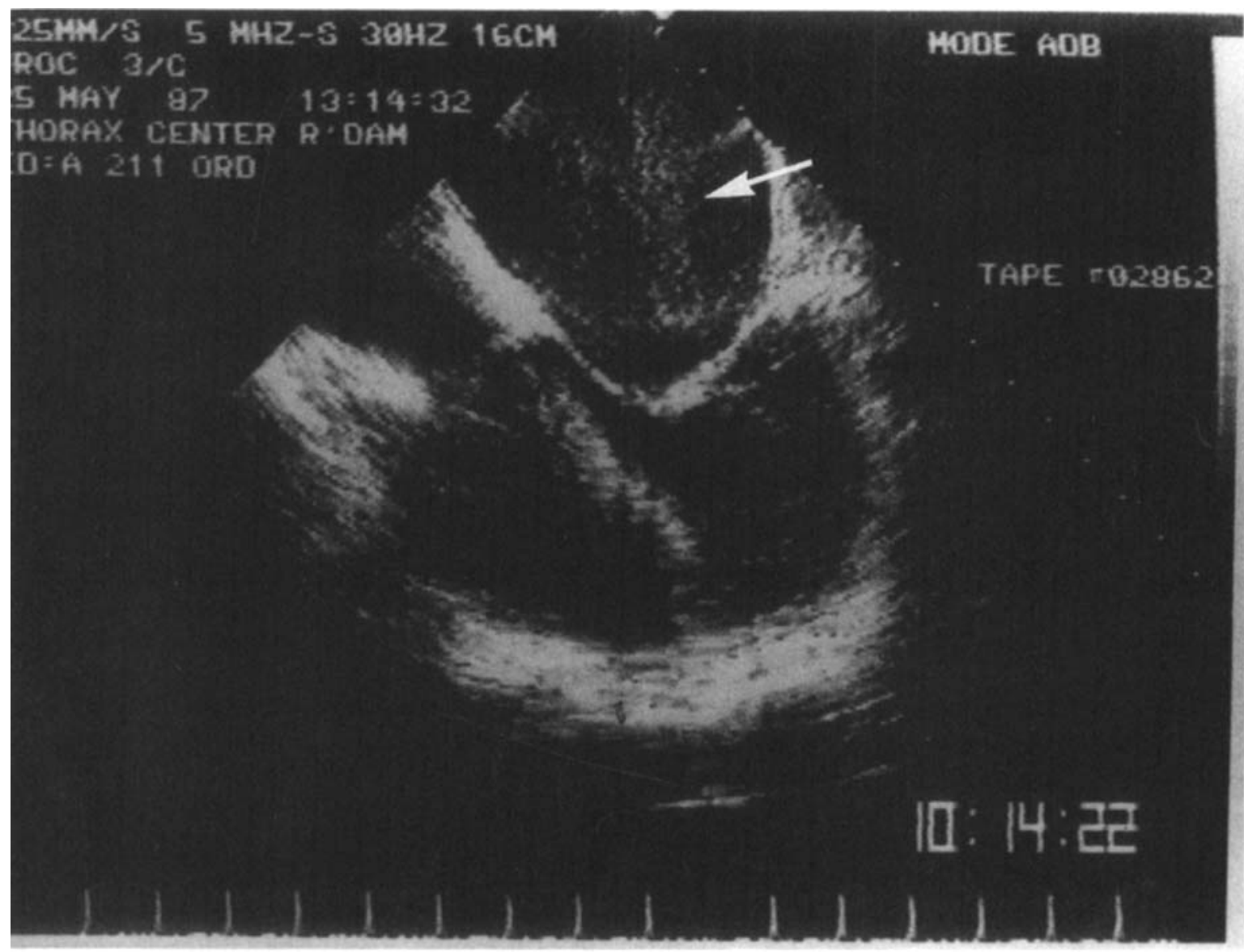

Figure 1. Demonstration of spontaneous contrast in patient 1 during transesophageal echocardiography prevalvuloplasty. 


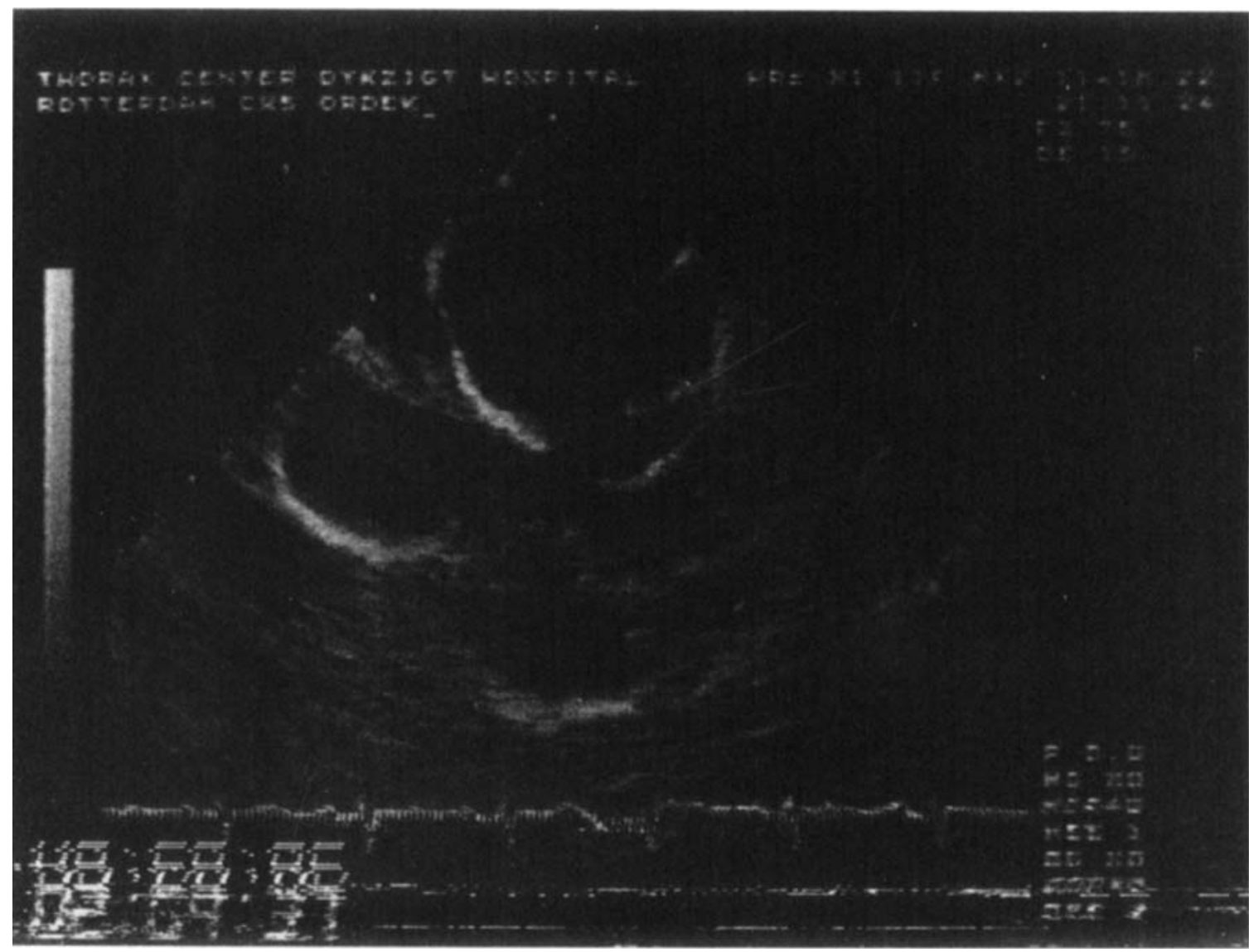

Figure 2. Absence of spontaneous echographic contrast in patient 1,6 months after balloon valvuloplasty.

Balloon valvuloplasty was preceded by a complete right and left heart catheterization and left ventricular angiography. Cardiac catheterization and transesophageal echocardiography were repeated 6 months after valvuloplasty.

In all three patients, a double-balloon technique was applied, using one conventional ( 10 or $15 \mathrm{~mm}$ ) and one Trefoil balloon $(3 \times 10$ or $3 \times 12 \mathrm{~mm})$. At the end of the procedure, the pressure gradient was measured, the valve orifice and the flow across the valve were calculated according to the Gorlin formula, and the degree of mitral regurgitation was assessed by means of the left ventricular angiogram. There were no thromboembolic complications.

Immediate results of the balloon valvuloplasty on hemodynamic parameters and transmitral velocities measured by precordial continuous wave echocardiography performed within hours of the procedure are shown in Table I.

Exercise tolerance according to NYHA classification improved from Class 2 or 3 to 1 . Spontaneous echocardiographic contrast was absent in patient 1 , 6 months after mitral valvuloplasty (Fig. 2) and is still present in the remaining two patients. In the latter ones, the transmitral flow, calculated at control catheterization, had decreased to the value before dilatation, while in patient 1 transmitral flow remained unchanged.

\section{Discussion}

Spontaneous echographic contrast on precordial echocardiography is known to be associated with low intracavitary flow, in the presence of left ven- 
tricular aneurysm, dilated cardiomyopathy, ${ }^{3}$ in the inferior vena cava in constrictive pericarditis, and in cases with aortic dissection spontaneous echoes may be seen within the false lumen of the aorta. ${ }^{4}$ Mikell et al. ${ }^{3}$ showed that routine formation of the erythrocytes are the source of blood echogenicity and not platelet aggregation. Blood within isolated vessels quickly become echogenic after the onset of stasis induced by cross-clamping maneuver.

This phenomenon has occasionally been noticed in the left atrium using precordial echocardiography in patients with mitral valve stenosis or after mitral valve replacement. ${ }^{5}$ Recently, Daniel et al. ${ }^{6}$ indicated the left atrial spontaneous contrast on transesophageal echocardiography, using a 3.5$\mathrm{MHz}$ transducer in patients with mitral stenosis or mitral valve replacement was accompanied by a higher incidence of left atrial thrombi and a higher risk for arterial thromboembolization. In two thirds of patients with moderate-to-severe mitral stenosis spontaneous contrast was diagnosed. In this study a $5.6-\mathrm{MHz}$ transducer was used, increasing the sensitivity for small particles producing back-scattering and easier visualization of spontaneous echographic contrast. ${ }^{2}$

Our three patients with moderate-to-severe mitral stenosis all had evidence of spontaneous echo contrast in the left atrium, using $5.6 \mathrm{MHz}$. None had a history of arterial embolization, while all were on anticoagulant therapy at the time of the examination. Six months after balloon valvuloplasty the phenomenon had disappeared in the pa- tient with persisting increased flow across the mitral valve. In the other two patients the calculated transmitral flow had decreased, while the spontaneous echographic left atrial contrast was still present. It is plausible that stasis of blood, which is responsible for its echogenicity, is induced by the slow transmitral flow in patients with mitral stenosis. Disappearance of spontaneous contrast after mitral valvuloplasty therefore appears to be a sign of increased transmitral blood flow and could be used as an indicator of sustained hemodynamic improvement.

\section{References}

1. Gussenhoven EJ, Taams MA, Roelandt JRTC. Transesophageal two-dimensional echocardiography: Its role in solving clinical problems. J Am Coll Cardiol 1986; 8:975979.

2. Taams MA, Gussenhoven EJ, Lancee CT. Left atrial vascularized thrombus diagnosed by transoesophageal cross sectional echocardiography. Br Heart J 1987; 58:669-671.

3. Mikell FL, Asinger RW, Elsberger J, et al. Regional stasis of blood in dysfunctional left ventricle: Echocardiographic detection and differentiation from early thrombosis. Circulation 1982; 66:755-763.

4. Panidis IP, Kotler MN, Mintz GS, et al. Intracavitary echoes in the aortic arch in type III aortic dissection. Am J Cardiol 1984; 54:1159-1160.

5. Beppu S, Nimura Y, Sakakibara H, et al. Smoke-like echo in the left atrial cavity in mitral valve disease: Its features and significance. J Am Coll Cardiol 1985; 6:744-749.

6. Daniel WG, Nellessen U, Schroder E, et al. Left atrial spontaneous echo contrast in mitral valve disease: $A n$ indicator for an increased thromboembolic risk. J Am Coll Cardiol 1988; 11:1204-1211. 